\title{
Remission of arthritis but persistent cutaneous lesions following tocilizumab treatment in a RA-patient suffering from concomitant psoriasis
}

\author{
Wolfgang Hartung ${ }^{1 *}$, Boris Ehrenstein ${ }^{1}$, Rotraud Wallisch ${ }^{1}$, Martin Fleck ${ }^{1,2}$ \\ ${ }^{1}$ Department of Rheumatology/Clinical Immunology, Asklepios-Hospital, Bad Abbach, Germany; \\ *Corresponding Author: w.hartung@asklepios.com, b.ehrenstein@asklepios.com, r.wallisch@asklepios.com \\ ${ }^{2}$ Department of Internal Medicine I, University of Regensburg, Regensburg, Germany; m.fleck@asklepios.com
}

Received 24 January 2013; revised 28 February 2013; accepted 10 March 2013

\begin{abstract}
Modulation of the interleukin-6 pathway with tocilizumab has been demonstrated to be highly effective in substantial numbers of patients suffering from rheumatoid arthritis (RA), and juvenile idiopathic arthritis. A pivotal role of the IL-6 pathway has been also established in other autoimmune conditions including psoriasis and psoriatic arthritis, becoming attractive targets for therapeutic IL-6 inhibition. Here, we describe the first case of tocilizumab treatment in a RApatient suffering from concomitant severe psoriasis, who achieved remission of RA, whereas no improvement of psoriatic lesions could be observed.
\end{abstract}

Keywords: Tocilizumab; Rheumatoid Arthritis; Psoriasis; Treatment

\section{INTRODUCTION}

Modulation of the interleukin-6 pathway with tocilizumab has been demonstrated to be highly effective in substantial numbers of patients suffering from rheumatoid arthritis (RA), and juvenile idiopathic arthritis. A pivotal role of the IL-6 pathway has been also established in other autoimmune conditions including psoriasis and psoriatic arthritis, becoming attractive targets for therapeutic IL-6 inhibition. In the following report we present the case of a woman treated with tocilizumab, simultaneously suffering from RA and psoriasis.

\section{CASE REPORT}

A 43-year-old woman with RA presented with high disease activity. The diagnosis of RA had been established 9 years previously, based on typical clinical disease manifestations, positive serology for rheumatoid factor, antinuclear antibodies, high titers of anti-citrullinated protein antibodies as well as radiographic findings characteristic of RA. Concomitantly, the patient suffered from severe plaque psoriasis, which had been confirmed by a dermatology specialist and treated topically. Since establishment of the diagnosis of RA, the patient had received several different combinations of DMARD's and biologic agents including MTX, cyclosporine, leflunomide, infliximab, etanercept, adalimumab, rituximab and abatacept. However, all of these treatments had to be discontinued either due to inefficacy or side effects. Off note, treatment with the TNF-inhibitors as well as abatacept did not result in relevant improvement of RA disease activity, and therefore patient presented with persistant high RA activity (DAS28 7,0) with numerous swollen joints and elevated systemic inflammatory parameters (ESR 53/109, CRP $48 \mathrm{mg} / \mathrm{l}$ ). Since modulation of the interleukin-6 pathway with tocilizumab has been demonstrated to be highly effective in substantial numbers of patients suffering from rheumatoid arthritis (RA), and juvenile idiopathic arthritis [1-6], and a pivotal role of the IL-6 pathway has been also established in other autoimmune conditions including psoriasis and psoriatic arthritis [7], treatment with tocilizumab had been initiated in combination with low-dose prednisolone. In addition, triamcinolone was injected in multiple affected joints. At that time, the patient presented with extensive cutaneous lesions owing to psoriasis (Figure 1A). After 12 weeks of tocilicumab treatment, a distinctive improvement of RA could be observed with a disease activity score within the remission range (DAS28 2.34), which had been not achieved with the previous therapeutic regimens. In addition, CRP and ESR were determined at normal levels. The beneficial effect of the tocilizumab treatment could be confirmed by musculoskeletal ultrasound of the right knee, demonstrating clear improvement of synovial hyperperfusion and effusion as well as reduction in the synovitis score (Figures 1C and D). However, there was 

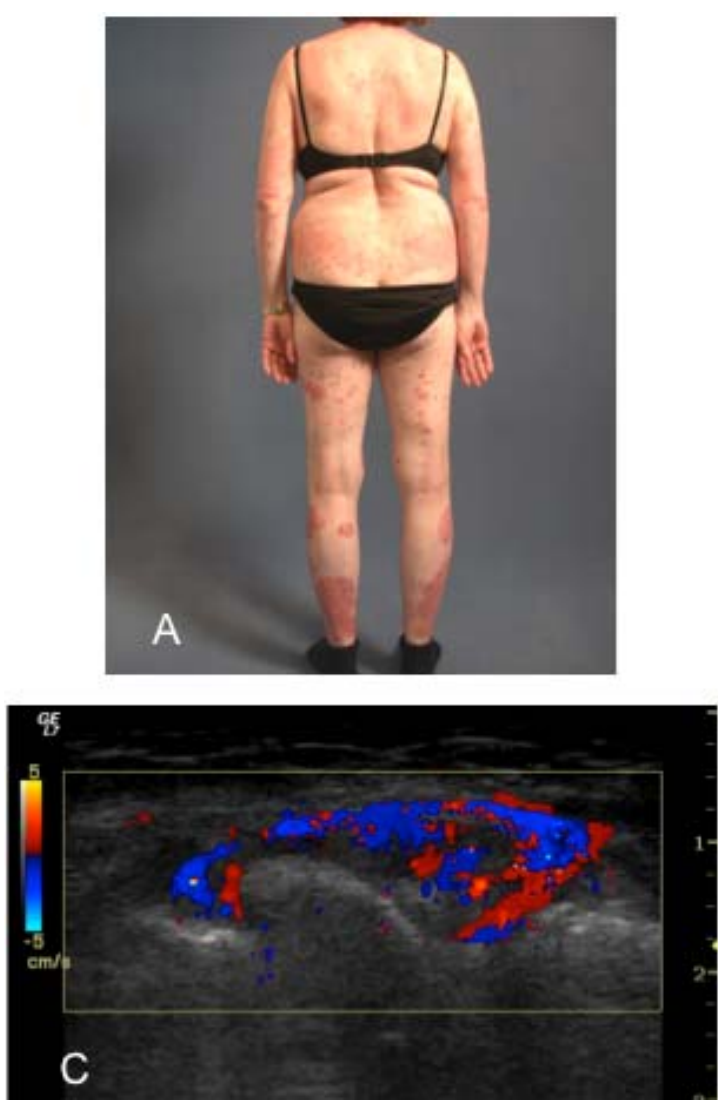
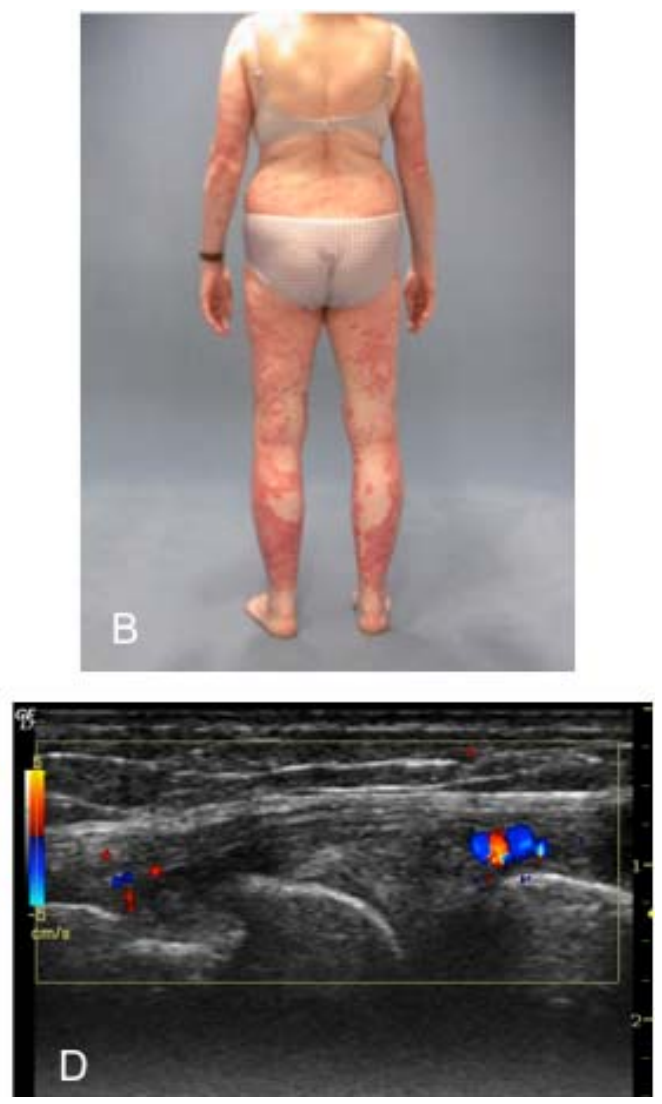

Figure 1. Due to refractory RA, the patient has been treated with tocilizumab. A: Concomitant psoriatic lesions before initiation of tocilizumab; B: Persistent psoriatic lesions 3 months following tocilizumab treatment; C: A severe synovitis of the right knee with hyperperfusion und effusion could be determined by muskuloskeletal ultrasound before tocilizumab treatment (Power-Doppler-Score: 3); D: Improvement of arthritis with minimal residual synovitis of the right knee could be detected by ultrasonography 3 months following initiation of tocilizumab treatment (Power-Doppler-Score: 1).

no improvement of the psoriatic lesions in this patient with a persistent extensive involvement of the whole integument, which appeared rather progressive (Figure 1B).

\section{DISCUSSION}

The patient demonstrated here was treated with tocilizumab in combination with low-dose prednisolone due to refractory RA. Multiple therapy regimens including several different DMARD's and biologic agents had failed to induce remission of RA, previously. In addition, the patient suffered from concomitant psoriasis with extensive cutaneous involvement. Following 3 months of tocilizumab treatment, complete remission of RA could be achieved for the first time after disease onset, demonstrating the pivotal role of the IL-6 pathway in the pathogenesis of RA in this patient. Since evidence indicates an important contribution of the IL-6 pathway also in the pathogenesis of psoriasis, tocilizumab treatment has been suggested as treatment option for psoriasis with and without concomitant psoriatic arthritis. In line with this consideration, it has been demonstrated that IL-6 can directly induce the production of IL-22 in human naive T cells, which mediates IL-23-induced acanthosis and dermal inflammation through the activation of Stat3 (signal transduction and activators of transcription 3) contributing to the formation of psoriatic skin lesions [8]. In addition, IL-6 has been attributed to the differentiation and activation of TH17 cells, critically involved in psoriasis [9]. However, there was no improvement of psoriasis-induced cutaneous lesions in the presented case despite achievement of RA remission following tocilizumab treatment. This observation suggests that the relevance of the IL-6 pathway to the pathogenesis and the underlying disease mechanisms might differ substantially between these two disease entities. Therefore, the therapeutic effect of IL-6 inhibition in psoriasis patients remains to be clarified.

\section{REFERENCES}

[1] Nishimoto, N., Yoshizaki, K., Miyasaka, N., Yamamoto, K., Kawai, S., Takeuchi, T., Hashimoto, J., Azuma, J. and 
Kishimoto, T. (2004) Treatment of rheumatoid arthritis with humanized anti-interleukin-6 receptor antibody: A multicenter, double-blind, placebo-controlled trial. Arthritis \& Rheumatism, 50, 1761-1769. doi:10.1002/art.20303

[2] Maini, R.N., Taylor, P.C., Szechinski, J., Pavelka, K., Bröll, J., Balint, G., Emery, P., Raemen, F., Petersen, J., Smolen, J., Thomson, D. and Kishimoto, T. (2006) Double-blind randomized controlled clinical trial of the interleukin-6 receptor antagonist, tocilizumab, in european patients with rheumatoid arthritis who had an incomplete response to methotrexate. Arthritis \& Rheumatism, 54, 2817-2829. doi:10.1002/art.22033

[3] Emery, P., Keystone, E., Tony, H.P., Cantagrel, A., van Vollenhoven, R., Sanchez, A., Alecock, E., Lee, J. and Kremer, J. (2008) Il-6 receptor inhibition with tocilizumab improves treatment outcomes in patients with rheumatoid arthritis refractory to anti-tumour necrosis factor biologicals: Results from a 24-week multicentre randomised placebo-controlled trial. Annals of the Rheumatic Diseases, 67, 1516-1523. doi:10.1136/ard.2008.092932

[4] Genovese, M.C., McKay, J.D., Nasonov, E.L., Mysler, E.F., da Silva, N.A., Alecock, E., Woodworth, T. and Gomez-Reino, J.J. (2008) Interleukin-6 receptor inhibition with tocilizumab reduces disease activity in rheumatoid arthritis with inadequate response to disease-modifying antirheumatic drugs: The tocilizumab in combination with traditional disease-modifying antirheumatic drug therapy study. Arthritis \& Rheumatism, 58, 2968-2980. $\underline{\text { doi: } 10.1002 / \text { art. } 23940}$
[5] Jones, G., Sebba, A., Gu, J., Lowenstein, M.B., Calvo, A., Gomez-Reino, J.J., Siri, D.A., Tomsic, M., Alecock, E., Woodworth, T. and Genovese, M.C. (2010) Comparison of tocilizumab monotherapy versus methotrexate monotherapy in patients with moderate to severe rheumatoid arthritis: The ambition study. Annals of the Rheumatic Diseases, 69, 88-96. doi:10.1136/ard.2008.105197

[6] Smolen, J.S., Beaulieu, A., Rubbert-Roth, A., Ramos-Remus, C., Rovensky, J., Alecock, E., Woodworth, T. and Alten, R. (2008) Effect of interleukin-6 receptor inhibition with tocilizumab in patients with rheumatoid arthritis (option study): A double-blind, placebo-controlled, randomised trial. Lancet, 371, 987-997. doi:10.1016/S0140-6736(08)60453-5

[7] Van Kuijk, A.W. and Tak, P.P. (2011) Synovitis in psoriatric arthritis: Immunohistochemistry, comparisons with rheumatoid arthritis, and effects of therapy. Current Rheumatology Reports, 13, 353-369. doi:10.1007/s11926-011-0181-y

[8] Zheng, Y., Danilenko, D.M., Valdez, P., Kasman, I., Eastham-Anderson, J., Wu, J. and Ouyang, W. (2007) Interleukin-22, a t(h)17 cytokine, mediates il-23-induced dermal inflammation and acanthosis. Nature, 445, 648-651. doi:10.1038/nature05505

[9] Annunziato, F., Cosmi, L., Liotta, F., Maggi, E. and Romagnani, S. (2009) Type $17 \mathrm{t}$ helper cells-origins, features and possible roles in rheumatic disease. Nature Reviews Rheumatology, 5, 325-331. doi:10.1038/nrrheum.2009.80 